\title{
Transformation to Small Cell Lung Cancer of Pulmonary Adenocarcinoma: Clinicopathologic Analysis of Six Cases
}

Soomin Ahn ${ }^{1}$ Soo Hyun Hwang Joungho $\mathrm{Han}^{1} \cdot$ Yoon-La Choi ${ }^{1}$ Se-Hoon Lee ${ }^{2} \cdot$ Jin Seok $\mathrm{Ahn}^{2}$ Keunchil Park ${ }^{2} \cdot$ Myung-Ju Ahn ${ }^{2}$ Woong-Yang Park ${ }^{3,4}$

${ }^{1}$ Department of Pathology and Translational Genomics, ${ }^{2}$ Division of Hematology and Oncology, Department of Medicine, ${ }^{3}$ Department of Molecular Cell Biology, Samsung Medical Center, Sungkyunkwan University School of Medicine, Seoul; ${ }^{4}$ Samsung Genomic Institute, Samsung Medical Center, Seoul, Korea

Received: February 24, 2016

Revised: April 6, 2016

Accepted: April 18, 2016

\section{Corresponding Author}

Joungho Han, MD

Department of Pathology and Translational

Genomics, Samsung Medical Center,

Sungkyunkwan University School of Medicine,

81 Irwon-ro, Gangnam-gu, Seoul 06351, Korea

Tel: +82-2-3410-2765

Fax: +82-2-3410-0025

E-mail: hanjho@skku.edu

\begin{abstract}
Background: Epidermal growth factor receptor (EGFR) tyrosine kinase inhibitors (TKIs) are considered the first line treatment for a subset of EGFR-mutated non-small cell lung cancer (NSCLC) patients. Although transformation to small cell lung cancer (SCLC) is one of the known mechanisms of resistance to EGFR TKIs, it is not certain whether transformation to SCLC is exclusively found as a mechanism of TKI resistance in EGFR-mutant tumors. Methods: We identified six patients with primary lung adenocarcinoma that showed transformation to SCLC on second biopsy $(n=401)$ during a 6 -year period. Clinicopathologic information was analyzed and EGFR mutation results were compared between initial and second biopsy samples. Results: Six patients showed transformation from adenocarcinoma to SCLC, of which four were pure SCLCs and two were combined adenocarcinoma and SCLCs. Clinically, four cases were EGFR-mutant tumors from non-smoking females who underwent TKI treatment, and the EGFR mutation was retained in the transformed SCLC tumors. The remaining two adenocarcinomas were EGFR wild-type, and one of these patients received EGFR TKI treatment. Conclusions: NSCLC can acquire a neuroendocrine phenotype with or without EGFR TKI treatment.
\end{abstract}

Key Words: Lung neoplasms; Receptor, epidermal growth factor; Tyrosine kinase inhibitor; Small cell lung carcinoma; Adenocarcinoma
Currently, lung cancer is classified into two broad histological subgroups: non-small-cell lung cancer (NSCLC) and small cell lung cancer (SCLC). The distinction between these two categories is important because the treatment options differ substantially. There are different chemotherapeutic regimens for SCLC and NSCLC, and the initial response to chemotherapy is much greater for patients with SCLC than for those with NSCLC., Currently, epidermal growth factor receptor (EGFR) tyrosine kinase inhibitors (TKIs) are considered the first-line treatment for a subset of EGFR-mutated NSCLC patients. ${ }^{3}$ In many cases, however, acquired resistance emerges within a year. ${ }^{4}$ Although the secondary T790M mutation has been well-described and reported in up to $60 \%$ of resistant samples, ${ }^{5}$ there have been several studies proposing histological transformation from
NSCLC to SCLC as another mechanism of EGFR TKI resistance. ${ }^{2,5-12}$ The possible explanation of this phenomenon can be the transformation of NSCLC, mostly adenocarcinoma (ADC), to high-grade neuroendocrine phenotype. ${ }^{2}$ The other possibility can be the presence of combined histology of NSCLC and SCLC in initial samples and acquisition of different histological areas in second biopsy samples. ${ }^{2}$ The reports showed that every transformed SCLC tumor sample retained its original EGFR-activating mutation, ${ }^{6-11}$ supporting the idea that these were not independent second-primary cancers. ${ }^{2}$ In addition, many patients with transformed SCLC tumors were female non-smokers, ${ }^{6-11}$ which is different from the typical SCLC patient demographic.

The recent reports of transformation from NSCLC to SCLC evoke questions regarding the origin of SCLC and clinical ques- 
tions. The rate of transformation to SCLC in TKI resistant tumors varied according to the study. ${ }^{5,6,10}$ Furthermore, it is not certain whether transformation to SCLC is exclusively found as a mechanism of TKI resistance in EGFR-mutant tumors. Practical questions include whether repeat biopsy is indicated after EGFR TKI resistance develops following treatment initiation, especially since a good response after switching to a SCLC chemotherapy regimen in transformed SCLC tumors has been reported. ${ }^{12}$

Here, we report six cases of SCLC transformed from pulmonary ADC in a single institute during a 6-year period.

\section{MATERIALS AND METHODS}

\section{Cases}

During a 6-year period (2010-2015), there were a total of 2,310 diagnoses of pulmonary ADC in our institute. Of 2,310 patients, 401 patients underwent a second biopsy or resection for recurrent or metastatic tumors. Out of 401 patients, a total of six patients (1.5\%) with primary lung ADC showed transformed SCLC morphology in second biopsy. Two experienced pathologists reviewed the histological slides (S.A and J.H). All patients were treated in the Department of Oncology, Samsung Medical Center (Seoul, Korea). Clinical and follow-up data were obtained through a retrospective analysis of the medical records, including age, sex, smoking history, treatment, clinical course and follow-ups. All patients were followed until March 2016 with median follow-up period of 39.2 months. The study was approved by the Institutional Review Board at Samsung Medical Center (2014-14-08610).

\section{EGFR mutation test}

DNA was extracted from sections of formalin-fixed, paraffinembedded (FFPE) tissue that was also used for histologic diag- nosis. Manual microdissection was performed if tumor cell percentages were less than $70 \%$ in available samples. Genomic DNA was extracted using Qiagen DNA FFPE Tissue Kit (Qiagen, Hilden, Germany) according to the manufacturer's instructions. In cases of lung ADC, routine testing for the EGFR mutation was performed in the pathology laboratory using peptide nucleic acid-mediated clamping polymerase chain reaction (PCR) mutation detection kit as previously described, ${ }^{13}$ and results were retrieved from electronic medical records. For one SCLC sample, the EGFR mutation was detected using targeted sequencing via Illumina HiSeq 2500 (Illumina Inc., San Diego, CA, USA), which was performed for clinical trial enrollment. For the rest of SCLC samples, the EGFR mutation was newly evaluated using Cobas test, a real-time PCR test as previously described. ${ }^{14}$ EGFR mutation results were available for all samples except for one that had no residual tumor.

\section{Immunohistochemistry}

In the current study, we used representative FFPE tissue sections for immunohistochemical staining (IHC). IHC for CD56 and thyroid transcription factor 1 (TTF-1) was performed for SCLC or combined tumors. Staining was performed on $3-\mu \mathrm{m}-$ thick sections from each case using a biotin-avidin-peroxidase method on a BOND-MAX autostainer (Leica, Wetzlar, Germany) after retrieval with T/E buffer (CD56) or citrate buffer (TTF-1). We used primary antibodies to CD56 (1:200, Novocastra, Newcastle upon Tyne, UK) and TTF-1 (1:100, Dako, Glostrup, Denmark).

\section{RESULTS}

\section{Sample information and histologic features}

Six patients showed transformation from ADC to SCLC.

Table 1. Sample information and pathologic features of six patients showing transformation from non-small-cell lung cancer to small cell lung cancer

\begin{tabular}{|c|c|c|c|c|c|c|c|c|c|}
\hline $\begin{array}{l}\text { Case } \\
\text { No. }\end{array}$ & $\begin{array}{l}\text { Initial } \\
\text { tumor }\end{array}$ & $\begin{array}{l}\text { Sample } \\
\text { type }\end{array}$ & $\begin{array}{c}\text { Sample } \\
\text { acquisition site }\end{array}$ & Subtype & $\begin{array}{c}\text { Interval } \\
\text { between } \\
\text { biopsy (mo) }\end{array}$ & Transformed tumor & $\begin{array}{l}\text { Sample } \\
\text { type }\end{array}$ & $\begin{array}{l}\text { Sample } \\
\text { acquisition } \\
\text { site }\end{array}$ & $\begin{array}{l}\text { IHC TTF-1/ } \\
\text { CD56 }\end{array}$ \\
\hline 1 & ADC & Biopsy & Lung & Acinar & 37 & SCLC & Biopsy & Celiac LN & $-/+$ \\
\hline 2 & $A D C$ & Biopsy & Lung, brain & Acinar and papillary & 21 & $\begin{array}{l}\text { Combined SCLC and } \\
\text { ADC }\end{array}$ & Biopsy & Lung & $-1+^{a}$ \\
\hline 3 & $A D C$ & Biopsy & LN 4 & Acinar & 8 & SCLC & Biopsy & LN 7 & $+/+$ \\
\hline 4 & ADC & Biopsy & Lung & Acinar & 5 & $\begin{array}{l}\text { Combined SCLC and } \\
\text { ADC }\end{array}$ & Biopsy & Lung (same site) & $+/ \mathrm{NA}$ \\
\hline 5 & ADC & Resection & Lung & Acinar & 31 & SCLC & Biopsy & Pleura & $+/+$ \\
\hline 6 & ADC & Resection & Lung & Acinar and solid & 50 & SCLC & Biopsy & Neck LN & $+/+$ \\
\hline
\end{tabular}

IHC, immunohistochemistry; TTF-1,thyroid transcription factor; ADC, adenocarcinoma; SCLC, small cell lung cancer; LN, lymph node; NA, not-applicable. ${ }^{a}$ CD56 was positive in only SCLC components. 

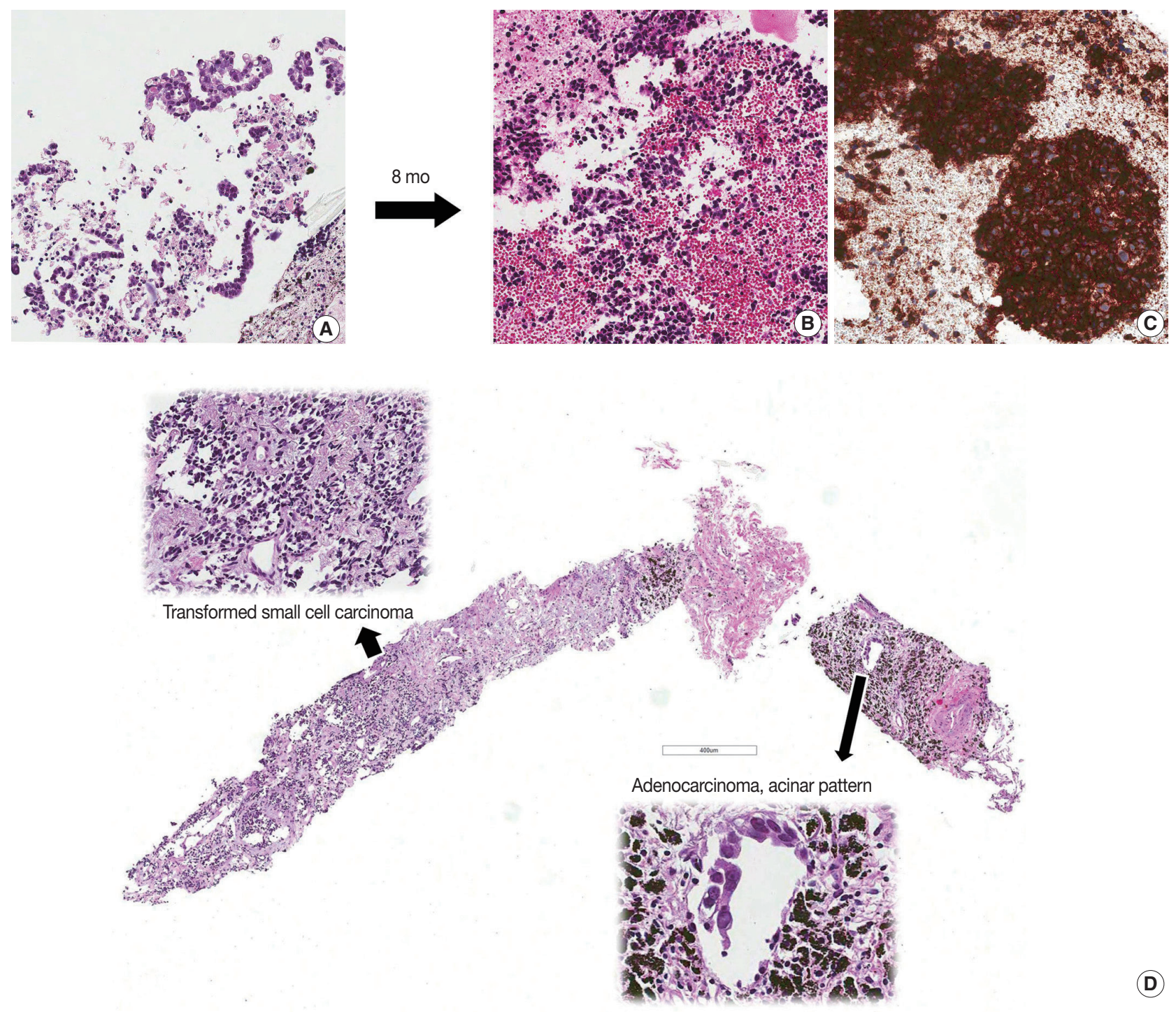

(D)
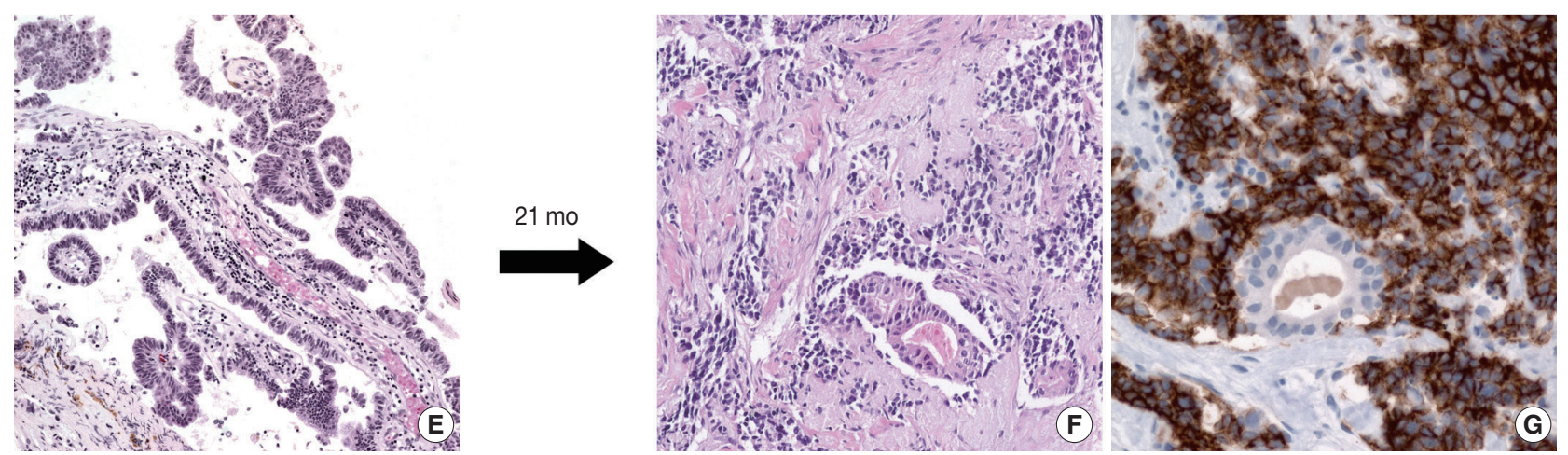

Fig. 1. Three cases showing transformation from non-small-cell lung cancer to small-cell lung cancer. (A) Initial biopsy of case 3 shows adenocarcinoma. Second biopsy after Iressa treatment, mediastinal lymph node specimen shows small cell carcinoma (B) and tumor cells are strongly positive for CD56 (C). (D) In case 4, second biopsy after gefetinib treatment reveals combined small-cell and adenocarcinoma histology. (E) Adenocarcinoma is identified in the brain tissue of case 2 at the time of initial diagnosis. (F) Second biopsy after afatinib treatment from this patient has combined small-cell and adenocarcinoma histology. (G) CD56 is expressed in the small cell component of the tumor sample. 
Sample information and pathologic features are summarized in Table 1. Of the initial samples with diagnosis of ADC, four were obtained using needle biopsy and two were surgically resected specimens. All second biopsies were obtained using needle biopsy. The histology of the six ADCs was acinar $(\mathrm{n}=4)$, mixed acinar and papillary $(\mathrm{n}=1)$, and mixed acinar and solid $(\mathrm{n}=1)$. Of samples that showed transformation to SCLC upon second biopsy, four showed pure SCLC morphology and two showed combined ADC and SCLC morphology. In two cases, ADC components demonstrated acinar morphology (Fig. 1). For small cell components, TTF- 1 was expressed in four of six cases and CD 56 was expressed in all five available cases. CD56 was not expressed in $\mathrm{ADC}$ components.

\section{Clinical information and EGFR status}

The clinical information and EGFR mutation status of the six patients are summarized in Table 2 . Initial treatments included complete resection and adjuvant chemotherapy for case 1 (cT2NO), palliative chemotherapy for case 2 (cT3N1M1) and case 4 (cT1N0M1), EGFR TKI for case 3 (cT2N3M1), incomplete resection and palliative chemotherapy for case 5 (cT1N0M1), and complete resection for case 6 (cT1NO). The sites of distant metastasis were as follows; brain (cases 2 and 4), pleura (case 3, 4, and 5), bone (case 4), and liver (case 4).

Of the six patients with ADC in initial biopsy, four (cases 1-4) harbored an EGFR mutation (L858R mutation, $\mathrm{n}=1$; exon 19 deletion, $\mathrm{n}=3$ ). All four, along with case 5 who had wild-type $E G F R$ but was enrolled in a clinical trial of gefetinib, were treated with EGFR TKIs. Cases 1 and 3 were treated with irressa, and afatinib was also added for case 1. Case 2 was treated with afatinib only, and cases 4 and 5 were treated with gefetinib only. All five patients who underwent TKI treatment were female non-smokers (Table 2). Despite TKI treatment, all five patients showed disease progression, upon which a second biopsy was performed. The interval between initial biopsy and second biopsy ranged from 5 to 50 months with mean of 25.3 months.

After confirmation of transformation of SCLC on second biopsy, four patients received further treatment. The treatment option for two patients (cases 1 and 3) was switched to etoposide and cisplatin, and one of them (case 3) showed partial response. Case 5 died due to disease progression and the other patients were alive in the short-term follow-up period.

EGFR mutation status was compared between initial and second samples, and all pairs showed the same EGFR status. The original EGFR mutation in cases $1-4$ was retained in all transformed SCLC samples, while cases 5 and 6 showed no

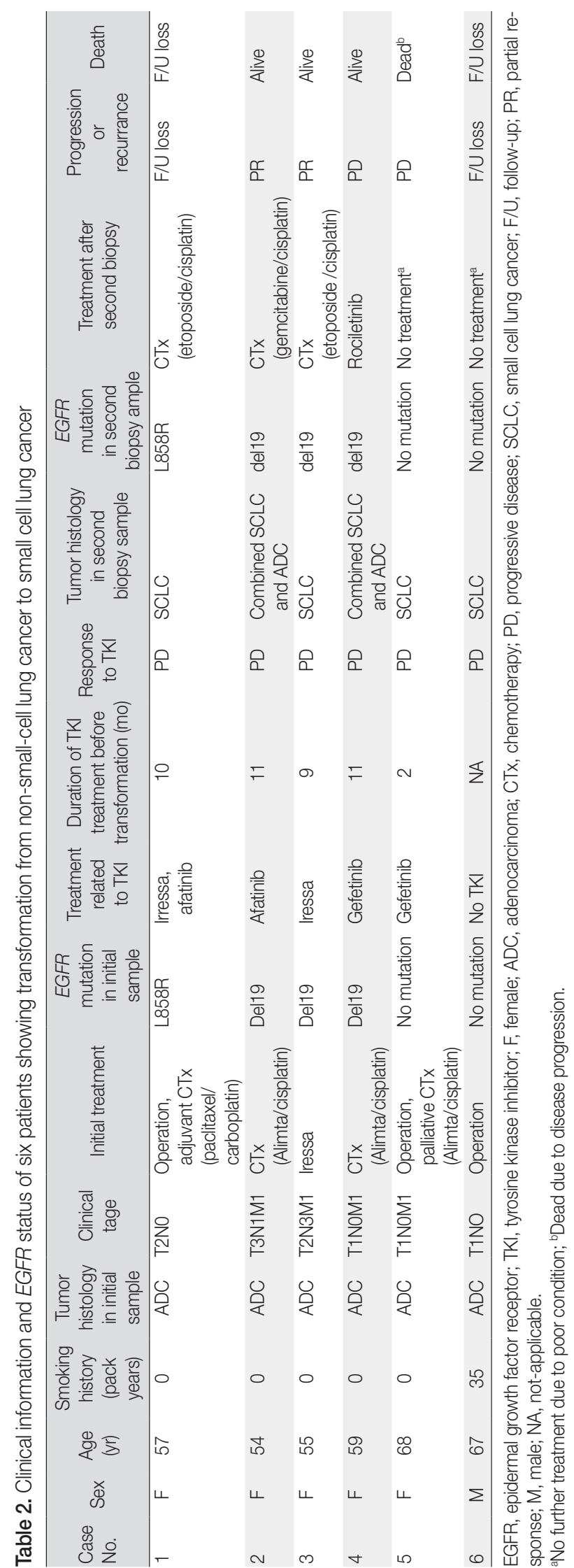


EGFR mutation in transformed SCLC samples.

\section{DISCUSSION}

Transformation of NSCLC to SCLC was recently proposed as a mechanism of resistance to TKI therapy. ${ }^{2,6}$ Identification of histologic transformation may be an important factor in determining a patient's treatment plan due to the differences between NSCLC and SCLC. While most reports of transformation of ADC to SCLC were identified in EGFR mutant patients related to TKI treatment, it is not certain whether transformation is exclusively related to EGFR mutation or EGFR TKI treatment. ${ }^{10}$ Here, we report six cases of ADC which showed histologic transformation to SCLC over a 6-year period at a single institute. Similar to previous reports, four cases in our series were ADC with EGFR activating mutations that underwent TKI treatment and were subsequently found to have SCLC transformation on second biopsy. However, we also identified two additional cases of SCLC transformation that had no EGFR mutation, and one of these cases underwent initial TKI treatment.

EGFR TKIs are now being used worldwide for first-line treatment in a subset of lung cancers bearing EGFR-activating mutations, and they have demonstrated dramatic therapeutic efficacy. ${ }^{15}$ However, acquired resistance through multiple mechanisms has become a major problem. ${ }^{6}$ One of mechanism of resistance to EGFR inhibitors is the histological transformation of ADC to SCLC. ${ }^{6}$ Although the presence of combined ADC and SCLC histology at initial diagnosis is a possibility, genomic sequencing of $E G F R$ mutations shows that both the original tumor and transformed SCLC at the time of resistance share the original EGFR-activating mutation, thus supporting the conclusion that these were not independent tumors. ${ }^{6-11}$ However, the small biopsy size represents only a portion of tumors, and SCLC components may become dominant at the time of disease progression. Of the six patients with initially diagnosed with ADC in our report, two were diagnosed using surgically resected samples rather than needle biopsies. For these two cases, the possibility of combined histology at the initial biopsy can be excluded. In case 6, it was difficult to distinguish between SCLC transformation and second primary SCLC considering the early stage of initial ADC.

In our series, two of six cases were $E G F R$-wild-type ADC. This suggests that transformation to SCLC is not unique to tumors bearing EGFR mutations, nor does it exclusively result from TKI treatment. Transformation to SCLC is also reported as a mechanism of acquired resistance to crizotinib in $A L K$ rearranged lung tumors. ${ }^{16}$ In addition, transformation to large cell neuroendocrine carcinoma was identified as an acquired resistance mechanism to EGFR TKIs and crizotinib. ${ }^{17,18}$ Recent studies suggest that alveolar type II cells can give rise to both ADC and SCLC, ${ }^{19}$ so EGFR-mutant lung cancers derived from alveolar type II cells may have the potential to transform into SCLC during the disease progression. ${ }^{2}$ In sum, it seems that acquisition of neuroendocrine phenotype, which includes SCLC transformation, can occur in the progression of disease in both $E G$ $F R$-mutant and $E G F R$-wild-type NSCLCs.

Second biopsies are not routinely performed for lung cancer patients when patients showed resistance to TKI treatment. Therefore, the incidence of transformation to SCLC in NSCLCs cannot be accurately calculated. An acquired TKI resistance arising from the histological transformation to SCLC has been reported to be as high as 3\%. In our institute, the incidence of transformation to SCLC identified in the second biopsy of total ADCs was $1.5 \%$. Recently in our institute, there were two cases of SCLC transformation in EGFR mutant ADC during treatment with AZD9291, an oral irreversible EGFR TKI with selectivity for activating EGFR mutations and the T790M resistance mutation. ${ }^{20}$ These cases were not included in this report. In clinical practice, identification of small cell component is important as the treatment option can be switched to etoposide and cisplatin against SCLC. ${ }^{12}$

In conclusion, we report six cases of lung cancer demonstrating transformation from ADC to SCLC. Four cases were EGFRmutant tumors from female non-smokers who underwent TKI treatment, and the EGFR mutation was retained in the transformed SCLC tumors. The other two ADCs were EGFR-wildtype, and one of these patients received EGFR TKI treatment. The neuroendocrine phenotype can thus be acquired during ADC disease progression independent of EGFR TKI treatment.

\section{Conflicts of Interest}

No potential conflict of interest relevant to this article was reported.

\section{REFERENCES}

1. Goldstraw P, Ball D, Jett JR, et al. Non-small-cell lung cancer. Lancet 2011; 378: 1727-40.

2. Oser MG, Niederst MJ, Sequist LV, Engelman JA. Transformation from non-small-cell lung cancer to small-cell lung cancer: molecu- 
lar drivers and cells of origin. Lancet Oncol 2015; 16: e165-72.

3. Moiseenko VM, Protsenko SA, Semenov II, et al. Effectiveness of gefitinib (Iressa) as first-line therapy for inoperable non-small-cell lung cancer with mutated EGFR gene (phase II study). Vopr Onkol 2010; 56: 20-3.

4. Engelman JA, Jänne PA. Mechanisms of acquired resistance to epidermal growth factor receptor tyrosine kinase inhibitors in nonsmall cell lung cancer. Clin Cancer Res 2008; 14: 2895-9.

5. Yu HA, Arcila ME, Rekhtman N, et al. Analysis of tumor specimens at the time of acquired resistance to EGFR-TKI therapy in 155 patients with EGFR-mutant lung cancers. Clin Cancer Res 2013; 19: 2240-7.

6. Sequist LV, Waltman BA, Dias-Santagata D, et al. Genotypic and histological evolution of lung cancers acquiring resistance to EGFR inhibitors. Sci Transl Med 2011; 3: 75ra26.

7. Zakowski MF, Ladanyi M, Kris MG; Memorial Sloan-Kettering Cancer Center Lung Cancer OncoGenome Group. EGFR mutations in small-cell lung cancers in patients who have never smoked. N Engl J Med 2006; 355: 213-5.

8. van Riel S, Thunnissen E, Heideman D, Smit EF, Biesma B. A patient with simultaneously appearing adenocarcinoma and smallcell lung carcinoma harbouring an identical EGFR exon 19 mutation. Ann Oncol 2012; 23: 3188-9.

9. Morinaga R, Okamoto I, Furuta K, et al. Sequential occurrence of non-small cell and small cell lung cancer with the same EGFR mutation. Lung Cancer 2007; 58: 411-3.

10. Norkowski E, Ghigna MR, Lacroix L, et al. Small-cell carcinoma in the setting of pulmonary adenocarcinoma: new insights in the era of molecular pathology. J Thorac Oncol 2013; 8: 1265-71.

11. Watanabe S, Sone T, Matsui T, et al. Transformation to small-cell lung cancer following treatment with EGFR tyrosine kinase inhibitors in a patient with lung adenocarcinoma. Lung Cancer 2013; 82: 370-2.
12. Kim WJ, Kim S, Choi H, et al. Histological transformation from non-small cell to small cell lung carcinoma after treatment with epidermal growth factor receptor-tyrosine kinase inhibitor. Thorac Cancer 2015; 6: 800-4.

13. Lee B, Han G, Kwon MJ, Han J, Choi YL. KRAS mutation detection in non-small cell lung cancer using a peptide nucleic acid-mediated polymerase chain reaction clamping method and comparative validation with next-generation sequencing. Korean J Pathol 2014; 48: 100-7.

14. Ahn S, Lee J, Sung JY, et al. Comparison of three BRAF mutation tests in formalin-fixed paraffin embedded clinical samples. Korean J Pathol 2013; 47: 348-54.

15. Pao W, Miller VA. Epidermal growth factor receptor mutations, small-molecule kinase inhibitors, and non-small-cell lung cancer: current knowledge and future directions. J Clin Oncol 2005; 23: 2556-68.

16. Miyamoto S, Ikushima S, Ono R, et al. Transformation to small-cell lung cancer as a mechanism of acquired resistance to crizotinib and alectinib. Jpn J Clin Oncol 2016; 46: 170-3.

17. Kogo M, Shimizu R, Uehara K, et al. Transformation to large cell neuroendocrine carcinoma as acquired resistance mechanism of EGFR tyrosine kinase inhibitor. Lung Cancer 2015; 90: 364-8.

18. Caumont C, Veillon R, Gros A, Laharanne E, Bégueret H, Merlio JP. Neuroendocrine phenotype as an acquired resistance mechanism in ALK-rearranged lung adenocarcinoma. Lung Cancer 2016; 92: 15-8.

19. Sutherland KD, Proost N, Brouns I, Adriaensen D, Song JY, Berns A. Cell of origin of small cell lung cancer: inactivation of Trp53 and $\mathrm{Rb} 1$ in distinct cell types of adult mouse lung. Cancer Cell 2011; 19: 754-64.

20. Ham JS, Kim S, Kim HK, et al. Two cases of small cell lung cancer transformation from EGFR mutant adenocarcinoma during AZD9291 treatment. J Thorac Oncol 2016; 11: e1-4. 